\title{
Ultra-thin Silicate Films on Metals
}

\author{
Shamil Shaikhutdinov* and Hans-Joachim Freund \\ Abteilung Chemische Physik, Fritz-Haber-Institut der Max-Planck-Gesellschaft, \\ Faradayweg 4-6, 14195 Berlin, Germany
}

\begin{abstract}
Silica is one of the key materials in many modern technological applications. "Surface science" approach for understanding surface chemistry on silica-based materials, on the one hand, and further miniaturization of new generation electronic devices, on the other, all these face the necessity of rational design of the ultrathin silica films on electrically conductive substrates. The review updates recent studies in this field. Despite the structural complexity and diversity of silica, substantial progress has recently been achieved in understanding of the atomic structure of truly two-dimensional silicates.
\end{abstract}

*Corresponding author: shaikhutdinov@fhi-berlin.mpg.de 


\section{Introduction}

Silica $\left(\mathrm{SiO}_{2}\right)$ and silicates as natural minerals constitute the largest part of the earth's crust [1]. Silica is one of the key materials in many modern technological applications and has been the subject of numerous studies related to microelectronics and catalysis [2-5]. Inspired by recent successful preparations of well-ordered thin silica films on metal single crystal surfaces [6], which are well-suited for a variety of "surface science" techniques, the ultrathin silica films may become interesting materials in their own rights in the growing family of truly two-dimensional materials such as graphene, silicene, etc. Despite yet unknown technologically relevant properties of such films, understanding of their atomic structures, controllable growth, and preparation with "atomic precision" may lead to rational design of the new generation metal-oxide-semiconductor transistors in nano-electronics [7]. In addition, the preparation of well-defined silica surfaces remains an important prerequisite for deeper understanding of surface reactions in geochemistry and catalysis over silica and related materials.

This topical review follows up our recent review papers $[6,8,9]$. Although we could not avoid some overlapping with the previous ones, here we updated experimental and theoretical studies on atomic structures and chemical properties of pure and substituted silica films. After a breakthrough in the year 2010, when the first preparation of bilayer silicate films on $\mathrm{Ru}(0001)$ has been reported [10], such films provided a template for preparation of the aluminosilicate films which mimic the outer surfaces of zeolites and may also serve as a benchmark for twodimensional zeolites [11]. Moreover, several families of hypothetical zeolites based on these silicate bilayers were proposed which raise the possibility of new, yet hypothetical materials with vanishingly low densities, extremely wide channels, and tunable pore diameter and shape [12]. Very recently, preparation of silicate films doped with transition metals like Fe resulted in systems which could be considered as the first "surface-science" model of clays [13]. Finally, single-layer silicate films turned out to bear some interesting structural similarities to graphene that allowed us to refer to them as "silicatene" [14].

The review is organized as follows. We first introduce the silica polymorphs and silicates having the sheet-like structural motif. Then silica films thermally grown on Si and SiC single crystals will briefly be discussed. Establishing the atomic structure of thin silica films on $\mathrm{Mo}(112)$ as a first successful example for the preparation of a well-defined, metal-supported silicate film, illustrates the crucial role of the interplay between experiment and theory in such studies. The bilayer silicate films in both pure and substituted forms will be discussed in more details, in particular with respect to creating suitable model systems for zeolites and clays. Hydroxylation of silicate surfaces by water adsorption are important steps in understanding of their surface chemistry. Finally, comparison of structural characteristics of a single-layer silicate ("silicatene") and graphene will be demonstrated for the films grown on Ru(0001). Very recent studies on silicatene-graphene hybrid structures will be highlighted. The review ends with some concluding remarks. 


\section{Silica and Silicates}

All known silica structures are made up from $\left[\mathrm{SiO}_{4}\right]$ tetrahedra. At atmospheric pressure, a phase diagram includes $\alpha$-quartz, $\beta$-quartz, tridymite, and $\beta$-cristobalite before it melts at $1705^{\circ} \mathrm{C}$ [4]. In turn, tridymite has a high number of additional phases [15]. Noteworthy, the high-temperature phases HP-tridymite and $\beta$-cristobalite consist of identical sheets of cornersharing $\mathrm{SiO}_{4}$ tetrahedra joined in a hexagonal close-packed or face cubic-centered arrangement, respectively [16], which resemble those in layered silicates. For structural classification and nomenclature of silicates one can refer to the excellent book of F. Liebau [1]. Here we only introduce sheet silicates, or phyllosilicates, which includes mica, serpentine, chlorite groups, as well as the clay minerals. In phyllosilicates, corner-sharing $\mathrm{SiO}_{4}$ tetrahedra form a "tetrahedral" (T) layer of six member rings, with all tetrahedra pointing towards "octahedral" (O) sheet of aluminum (gibbsite) or magnesium hydroxide (brucite). Mg occupies all octahedral sites, while Al will only occupy 2 out of every 3 , thus giving rise to two groups of sheet silicates: the trioctahedral silicate, where each $\mathrm{O}$ or $\mathrm{OH}$ ion is surrounded by three divalent cations, like $\mathrm{Mg}^{2+}$ or $\mathrm{Fe}^{2+}$, and the dioctahedral one, where each $\mathrm{O}$ or $\mathrm{OH}$ ion is surrounded by two trivalent cations as $\mathrm{Al}^{3+}$. The T-O-T sheets are then connected to each other by a layer of cations, which is weakly bonded and often have water and other molecules trapped between the sheets. In addition, clays as layered aluminosilicates may be formed by T-O sheets in the 1:1 structure, or T-O-T sheets in the 2:1 structure. These structures are schematically shown in Fig. 1.

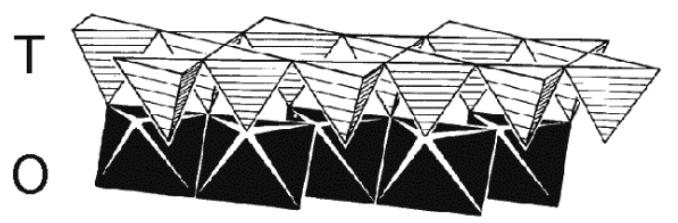

a)

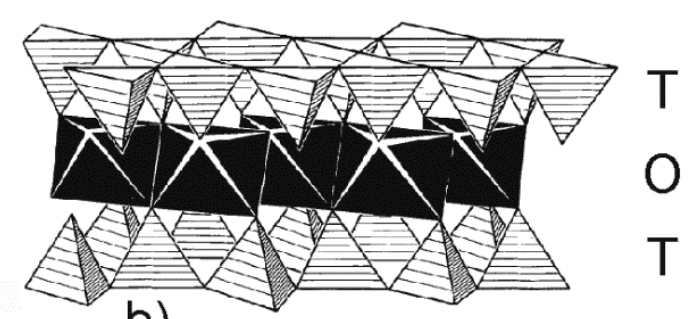

b)

Figure 1. Stacking of tetrahedral (T-) and octahedral (O-) layers in micas and clay minerals. Ideal kaolinite-like (a), and talc-like (or mica-like) (b) sheets. Adapted with permission from ref.[1]. Copyright (1985). Springer-Verlag Berlin Heidelberg.

Due to insulating properties of silica and silicates, experimentally available structural information about their surface structures is really scarce. This situation brought researchers to perform computational studies. Amongst the crystallographic forms of $\mathrm{SiO}_{2}$, the (001) surface of $\alpha$-quartz has often been addressed as it represents the most stable quartz surface at ambient conditions and possesses important common characteristics of silicas. A first-principles molecular dynamics study of the $\alpha$-quartz(001) surface showed that the surface is strongly 
reconstructed [17]. The most favorable reconstruction revealed an unexpected densification of the uppermost layers with three-membered and six-membered rings which do not exist in bulk quartz. Further periodic density functional theory (DFT) calculations showed that such a reconstruction distorts at least the top six layers of atoms and, consequently, the calculated surface energies critically depend on the depth of the reconstructed surface [18]. Apparently, hybrid functionals predicted energies more accurately. The reconstructed surfaces are shown in Fig. 2. Further structural optimization has recently been reported that used Born-Oppenheimer molecular dynamics simulations and DFT [19].
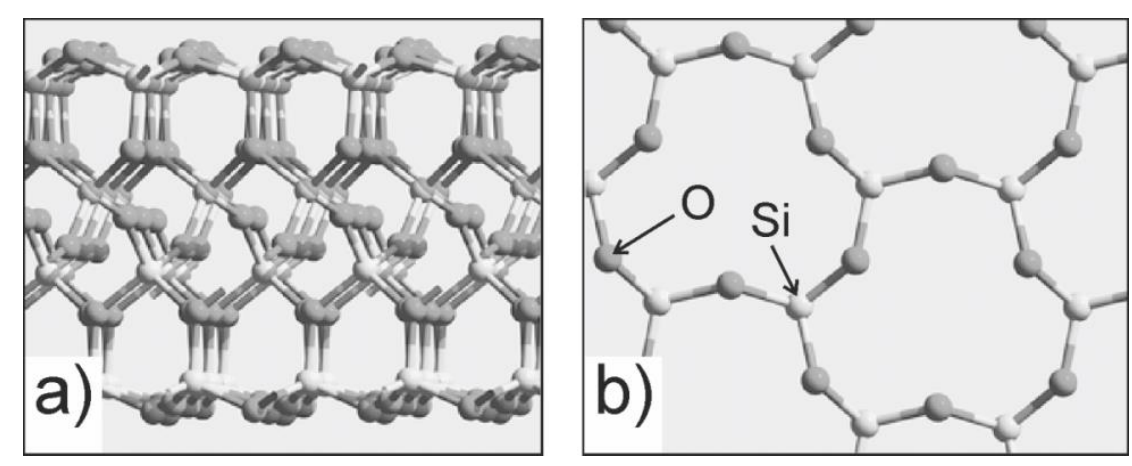

Figure 2. (a) Side view of the reconstructed $\alpha$-quartz (001) surface as predicted by DFT. (b) Six-membered rings formed at surface are shown in (b) depicting only topmost $\mathrm{Si}$ and $\mathrm{O}$ atoms for clarity. Adapted from ref. [18] with permission from the PCCP Owner Societies.

\section{Silica thin films on Si and SiC single crystals}

Driven by the key role of $\mathrm{SiO}_{2} / \mathrm{Si}$ interface in microelectronics, thermal growth of silica films on Si single crystal surfaces has extensively been studied, focusing on the structural and electronic properties of resulted silica overlayers as well as on the mechanism and kinetics of the oxide film growth $[3,20,21]$. In principle, the initial stages of oxidation fit well the so-called Cabrera-Mott mechanism [22] of oxygen diffusion through the oxide layer driven by a surface electric field. It is generally accepted that the bulk of silica films grown on Si crystals has the structure of amorphous (vitreous) silica. The structure of the interface between $\mathrm{SiO}_{2}$ and crystalline Si remains controversial. It appears that the transition region depends on the $\mathrm{Si}$ crystal orientation and oxidation agent, and is less than $0.5 \mathrm{~nm}$ in thickness [20, 23-27]. Although the $\mathrm{SiO}_{2} / \mathrm{Si}(100)$ interface could recently be atomically resolved by aberrationcorrected high-resolution transmission electron microscopy (TEM) in a cross-sectional mode [28] (see also TEM studies in refs. [27, 29, 30]), the thinnest silica films grown on Si crystals are, in essence, poorly defined. In addition, TEM studies revealed non-uniform structure along the interface. Therefore, several structural models have been considered [31] including silicon 
dangling bonds and hydrogen species (Fig. 3). Such imperfections largely contribute to the enhanced leakage current through the interface [32] that certainly impedes practical use of ultrathin silica films in electronic devices. Recent molecular dynamic simulations [33] showed that the interface between $\mathrm{Si}(100)$ and $\beta$-cristobalite exhibits an amorphous arrangement of atoms. In all other computed interfaces, the crystalline order remained, with defects in form of dislocated oxygen atoms. The interfaces between silicon and tridymite were found as the most ordered and energetically the most stable, albeit with the highest strain in the silicon layer.

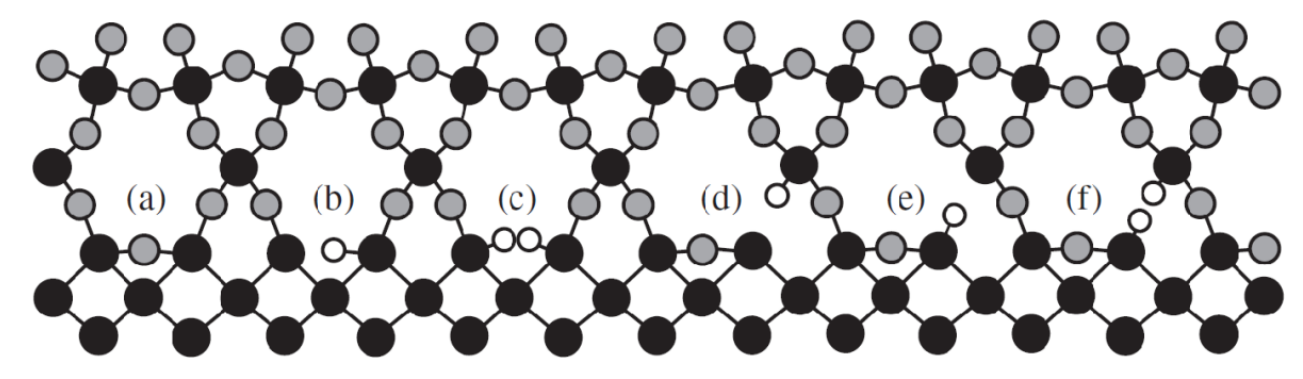

Figure 3. Several structural models of $\mathrm{Si} / \mathrm{SiO}_{2}$ interface [31]. Perfect interface without any defects (a); interface where an oxygen atom on the interface layer is replaced by a hydrogen atom (b); interface where an oxygen atom on the interface layer is replaced by two hydrogen atoms (c); interface where an oxygen atom on the second interface layer is replaced by a hydrogen atom and the lower silicon atom has a dangling bond (d); interface where an oxygen atom on the second interface layer is replaced by a hydrogen atom and the upper silicon atom has a dangling bond (e); and interface where an oxygen atom on the second interface layer is replaced by two hydrogen atoms (f).

Certainly, the lack of ordering at the interface can be rationalized in terms of a large lattice mismatch between $\mathrm{SiO}_{2}$ and $\mathrm{Si}$. In this respect, it has been recognized that lattice parameter of $\mathrm{SiO}_{2}$ matches fairly well that of silicon carbide ( $\mathrm{SiC}$ ), which also forms a passivating silica film in oxygen ambient. Note that the formation of a high quality insulating oxide layer on $\mathrm{SiC}$ is a key requirement for metal-oxide-semiconductor devices based on this material [34]. Recent review [35] summarizes the most important information about the structural aspects of silicon carbide, and $\mathrm{SiC} / \mathrm{SiO}_{2}$ interfaces and discusses kinetics of the processes involved. Again, as in the case of $\mathrm{SiO}_{2} / \mathrm{Si}$, many interfacial structures are, in principle, possible. In the course of such studies, well-ordered silica overlayers were unexpectedly observed by exposing the $\mathrm{H}$ etched $\mathrm{SiC}(000-1)$ surface to air without any further treatment [36]. Even after leaving a sample in air for several hours the well-ordered phase remained at the surface. The surface displayed a sharp $(\sqrt{ } 3 \times \sqrt{ } 3) R 30^{\circ}$ low energy electron diffraction (LEED) pattern. On the basis of dynamical LEED analysis, two structural models containing a $\mathrm{Si}_{2} \mathrm{O}_{3}$ layer on the C-terminated, $\mathrm{SiC}(000-1)$ surface and $\mathrm{Si}_{2} \mathrm{O}_{5}$ layer on the Si-terminated, $\mathrm{SiC}(0001)$ surface were proposed (Fig. 4), which were further validated by the DFT calculations [37]. 


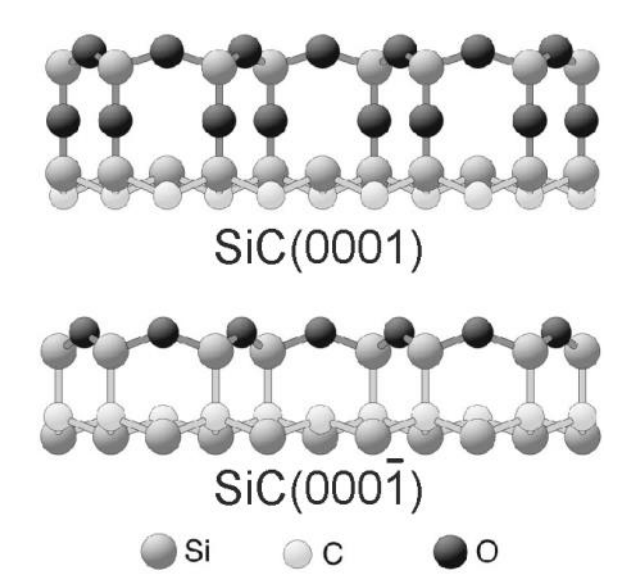

Figure 4. Cross views of silica layers formed on the C- and Si-terminated SiC surfaces. Adapted with permission from ref. [36]. Copyright (1999). AIP publishing LLC.

\section{Single-layer silicate films on metals}

To understand the principal structures and structural motif of the ultrathin silica films on metals, it is instructive here to recall the key experimental findings, which in combination with DFT calculations allowed one to determine the atomic structure of a crystalline silica film on $\mathrm{Mo}(112)$ [38, 39]. Although metal supported thin silica films were first introduced by Goodman and co-workers on Mo(110) and Mo(100) [40-42], the films only showed high degree of crystallinity when grown on a Mo(112) substrate $[43,44]$. The preparation includes exposure of the clean $\mathrm{Mo}(112)$ surface to oxygen to form the $\mathrm{p}(2 \times 3) \mathrm{O}-\mathrm{Mo}(112)$ surface, followed by physical vapour deposition (PVD) of Si and annealing in ultra-high vacuum (UHV) at high temperatures $(\sim 1250 \mathrm{~K})$. The resulted films exhibited a $c(2 \times 2)$ symmetry with respect to Mo(112) which may, in fact, be considered as slightly distorted hexagonal structure with the averaged unit cell length of $\sim 5.2 \AA$. Indeed, a honeycomb-like structure with a $\sim 5.4 \AA$ periodicity was observed by scanning tunneling microscopy (STM) [38, 45]. An x-ray photoelectron spectroscopy (XPS) study showed Si solely in the 4+ oxidation state with the binding energy (BE) of the Si $2 p$ core level at $\sim 103 \mathrm{eV}$, whereas two different chemical environments for oxygen were found, with BE of the $\mathrm{O} 1 \mathrm{~s}$ level at $532.5 \mathrm{eV}$ and $531.3 \mathrm{eV}$ and their intensity ratio about 3:2, thus associated with Si-O-Si and Si-O-Mo species [39, 46]. Finally, an infrared reflection-absorption spectroscopy (IRAS) measurements revealed a strong band at $\sim 1050 \mathrm{~cm}^{-1}$ (depending on annealing temperature $[38,43]$ ) assigned to the Si-O-Mo linkages [47]. This principal as well as other, much more weaker IRA bands (at $\sim 770$ and $\sim 675 \mathrm{~cm}^{-1}$ ) were red-shifted by 41,7 and $19 \mathrm{~cm}^{-1}$, respectively, on the films prepared with ${ }^{18} \mathrm{O}_{2}$ isotope [38]. 
The experimental results provided a solid basis for structural modeling with DFT. Two groups $[38,48]$ independently came to the conclusion that the structure of a silica film on Mo(112) must be considered as a two-dimensional (2D), single-layer network of corner-sharing $\left[\mathrm{SiO}_{4}\right]$ tetrahedra with one corner of each tetrahedron forming a Si-O-Mo bond (Fig. 5a). Such a structural motif was originally considered [47], but rejected, by Goodman's group which put forward the model of isolated $\left[\mathrm{SiO}_{4}\right]$ clusters arranged in $\mathrm{c}(2 \times 2)$ manner on $\mathrm{Mo}(112)$ [49]. Our group found the latter model to be inconsistent with experimental results, in particular with IRA-spectra. Later, further support for the 2D-network model came from the fast atom scattering [50] and LEED studies [51].

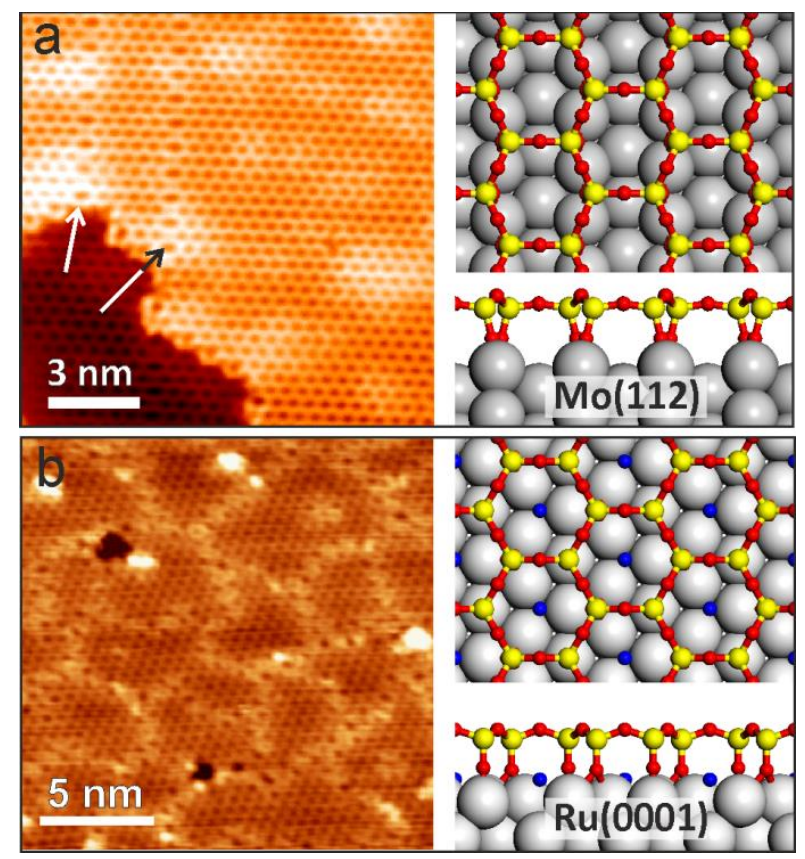

Figure 5. High resolution STM images and structural models (in top and cross views) of monolayer silicate films grown on Mo(112) (a) and Ru(0001) (b). Si (yellow), O (in red). Marked in blue are the $\mathrm{O}$ atoms directly bonded to the metal surface atoms in so called "O-rich" structure, shown here only for a Ru(0001) substrate. The arrows over STM image in panel (a) indicate anti-phase domain boundaries, consisting of alternating 8- and 4-membered rings.

The DFT results fully agreed with all experimental findings and, in particular, with the IRAS results which, as it has turned out, provide the most crucial information about the principal structure of silica films on metals (see below). Basically, three vibrational modes were computed, which are only detectable by IRAS due to the well-known metal selection rules: The band at $1060 \mathrm{~cm}^{-1}$ originates from asymmetric stretching of the Si-O bond pointing to the Mo substrate; the band at $778 \mathrm{~cm}^{-1}$ is assigned to the Si-O-Si symmetric stretching coupled with Si$\mathrm{O}-\mathrm{Si}$ bending, and the band at $670 \mathrm{~cm}^{-1}$ is resulted from a coupling of Si-O-Si bending modes. A very detailed analysis of the vibrational spectra based on symmetry considerations was 
provided in ref. [39] in order to explain, how these spectra relate to the transverse and longitudinal vibrational modes measured on the bulk-like systems, e.g. "thick" silica films and single crystals. In addition, the DFT results predicted the presence of additional $O$ atoms bonded only to the Mo atoms in the so-called "O-rich" films, which were then observed experimentally [52].

The structural model shown in Fig. $5 a$ is, in fact, nothing else, but a T-layer in sheet silicates (see Fig. 1) chemisorbed on a metal surface via apical oxygen atoms otherwise unsaturated. To recall, a similar, 6-membered rings termination was predicted by DFT as the most stable for the reconstructed $\alpha$-quartz(001) surface (Fig. 2) [17], yet not proven experimentally. Again, such a silicate layer was observed on SiC crystal surfaces (Fig. 4) [37]. In the latter case, a hexagonal $(\sqrt{ } 3 \times \sqrt{ } 3) R 30^{\circ}$ structure is observed that corresponds to a lattice constant $5.25 \AA$, which is close to $5.3 \AA$ computed for a free standing silicate layer [53]. In the first approximation, such a hexagonal silicate sheet would hardly fit the Mo(112) surface, having a rectangular unit cell $(5.46 \AA \times 8.92 \AA)$, causing a silicate lattice extension along the Mo[-1-11] direction to $5.46 \AA$ [38]. Nonetheless, the monolayer silica films on $\mathrm{Mo}(112)$ are wellordered on a large scale and form wide terraces, with only few anti-phase domain boundary defects running along Mo[-110] (Fig. 5a). Apparently, the main reason is a strong O-Mo bond in the Si-O-Mo linkages that stabilizes such a distorted structure. It is this bonding that governs also the formation of one-dimensional (1D) silica structures at sub-monolayer coverages [52, 54].

The situation considerably changes upon using metal surfaces with a lower affinity for oxygen, that is $\mathrm{Ru}(0001)$ in our case. Combined STM, XPS, and IRAS results, corroborated by DFT calculations, showed again, that a single silicate sheet grows on $\mathrm{Ru}(0001)$ under certain conditions (Fig. 5b) [55]. The preparation includes PVD of $0.5 \mathrm{ML}$ (with respect to Ru(0001) of Si onto the $30(2 \times 2)-\mathrm{Ru}(0001)$ surface at $\sim 100 \mathrm{~K}$ in oxygen ambient, followed by high-temperature annealing $(\sim 1200 \mathrm{~K})$ in $\sim 10^{-6}$ mbar of $\mathrm{O}_{2}$.

The IRA-spectra of the resulted films feature the principal band at $1134 \mathrm{~cm}^{-1}$ and much weaker signals at 790 and $687 \mathrm{~cm}^{-1}$, clearly resembling ones observed for $\mathrm{SiO}_{2.5} / \mathrm{Mo}(112)$ films (i.e. 1060, 778, and $670 \mathrm{~cm}^{-1}$ ). In addition, the band at $1074 \mathrm{~cm}^{-1}$ appears under some preparation conditions. The STM images revealed an atomically flat, honeycomb-like surface with a $\sim 5.4 \AA$ periodicity, in full agreement with a (2×2)-Ru(0001) LEED pattern. However, in contrast to the case of the rectangular $\mathrm{Mo(112)}$ surface supporting almost a perfect silicate layer, the films on $\mathrm{Ru}(0001)$ exhibited domains of 5-10 nm in size, thus producing a network of domain boundaries (Fig. 5b). This finding is consistent with the above-mentioned view on the role of Si-O-Metal bond in stabilizing the silicate layer on metal such that the stronger bond results in the better ordered film despite the lattice mismatch.

The DFT calculations fully supported the monolayer structure on $\mathrm{Ru}(0001)$ [55]. The simulated IRA spectrum showed the most intense IRA-active mode at $\sim 1160 \mathrm{~cm}^{-1}$, assigned to 
the in-phase combination of asymmetric stretching vibrations of the Si-O-Ru linkages. The weak signal at $1076 \mathrm{~cm}^{-1}$ is due to symmetric O-Si-O stretching vibrations. The bands at 820 and 677 $\mathrm{cm}^{-1}$ are assigned to the combinations of asymmetric stretching of Si-O-Ru linkages and O-Si-O bending modes. In addition, the calculations explained XPS spectra obtained for this structure [55].

Furthermore, the silicate films were prepared on the Pt(111) surface, which has a much lower affinity for oxygen than $\mathrm{Ru}(0001)$ [56]. The film preparation includes basically the same recipe as used for $\mathrm{Ru}(0001)$. However, the results only showed the formation of bilayer films (see next section) suggesting that monolayer films on $\mathrm{Mo}(112)$ and $\mathrm{Ru}(0001)$ are stabilized by strong Si-O-Metal bonds which are definitely weaker in the case of $\mathrm{Pt}(111)$. Note, on the other hand, that $\mathrm{Pt}(111)$ has a slightly larger lattice constant than Ru(0001), i.e. 2.77 and $2.71 \AA$, respectively, thus resulting in a larger lattice mismatch between silicate and $\mathrm{Pt}(111)$ when compared to Ru(0001).

Very recently, Banhart, Krasheninnikov and co-workers employed a "solid state" growth of silica overlayers on metals in an electron microscope, i.e. in the same manner as they previously used for graphene $[57,58]$. Silica covered TEM grids were first annealed in air at $350^{\circ} \mathrm{C}$ to form $\sim 10 \mathrm{~nm}$-thick, amorphous $\mathrm{SiO}_{2}$ films. Different metals (Fe, Co, Ru) were then deposited by PVD to about $5 \mathrm{~nm}$ in nominal thickness. The grids were transferred to a heating stage in a TEM to monitor structural transformations in situ. Annealing at $700^{\circ} \mathrm{C}$ in the microscope (a background pressure was in the range of $10^{-7} \mathrm{mbar}$ ) resulted in crystalline platelets of Fe and Co with a thickness of approximately $10 \mathrm{~nm}$. A few minutes after cooling the specimen to $450^{\circ} \mathrm{C}$, the nucleation and growth of a honeycomb-like silica films on the metal surface have been found. Figure 6 displays the cross-view TEM image of a silica film formed on a Fe particle which, on the basis of image simulations, was assigned to a monolayer silicate film.

The authors explained silica growth by bulk rather than surface diffusion of $\mathrm{Si}$ and $\mathrm{O}$ atoms through the metal layers, since preferential nucleation was observed on grain boundaries, where the diffusion of $\mathrm{Si}$ atoms is likely more facile. Seemingly, metal particles dissolve $\mathrm{Si}$ (and $\mathrm{O}$ ) atoms upon first heating to $700^{\circ} \mathrm{C}$ which then precipitate upon cooling as silicate layer. Interestingly, for the formation of silica layer, a clean metal surface was always necessary so that an intense "beam shower" had to be applied on a selected area of the metal prior to growth. (The silica overlayers on metals were sensitive to the $200 \mathrm{keV}$ electron beam and disappeared under intense irradiation). 


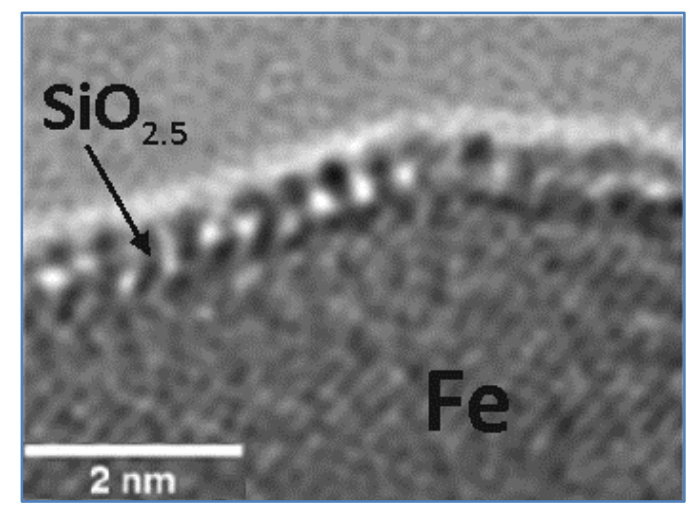

Figure 6. Cross-sectional TEM image of a silica single-layer film grown at $470^{\circ} \mathrm{C}$ on Fe particles deposited onto amorphous silica. Adapted with permission from ref. [57]. Copyright (2013). American Chemical Society.

A following-up study [58] of the same group revealed the formation of 1D silica structures, which followed the symmetry of the underlying metal surface, prior to the growth of the 2D monolayer films. The TEM observations were assigned to 1D silica decorating step edges of terraces. The authors reasonably assumed that surface steps act as pinning sites for migrating $\mathrm{Si}$ and $\mathrm{O}$ atoms which react towards silica. Several models, which are considerably different from those previously reported for 1D-silica structures observed on Mo(112) [54], were investigated by DFT. The calculations showed that 1D silica is energetically favorable over 2D structures if surface steps prevail on the substrate.

\section{Double-layer silicate films}

All attempts to prepare multilayer films on $\mathrm{Mo(112)}$ in a layer-by-layer mode only resulted in poorly-defined, amorphous silica films, albeit having smooth surface and sharp phonon spectra of bulk-like silica. Further annealing of the "as prepared" thick films at higher temperatures in attempt to make films better ordered caused either film decomposition or led back to a single-layer film, thus indicating a "self-limiting" growth of a crystalline silicate layer on metals like Mo. Another approach to suppress the formation of the very strong Si-O-Mo bonds, which apparently govern the formation of single layer structures, by the initial formation of the Si-Mo interface and subsequent oxidation was not successful, either [59]. Therefore, other metal substrates had to be addressed, in particular those having a lower affinity to oxygen. In this respect, Ru(0001) and Pt(111) were considered as good candidates, as both surfaces are often used for preparation of thin oxide films [60].

As already discussed in the previous section, preparation of silica films on $\mathrm{Ru}(0001)$ at low Si coverage leads to single layer structures. The most striking change in structural characteristics of the films, obtained by doubling the amount of Si deposited, is appearance of very sharp and intense IRA bands at 1300 and $692 \mathrm{~cm}^{-1}$ and, concomitantly, disappearance of 
the bands associated with the monolayer structure. Such infrared bands have never been reported for silica polymorphs and thermally grown silica films on Si. Analysis of possible structures, previously considered in DFT calculations for films grown on Mo(112) [45], suggested that, in this case, a double-layer structure could be formed, which is composed of two silicate layers linked together through apical $O$ atoms forming a mirror plane. From the chemical wisdom, such a structure terminated by fully saturated oxygen layer on either side must be weakly bound to the supporting metal surface. Therefore, double-layer models had to be computed by DFT including semi-empirical dispersion correction [10]. The adhesion energy was calculated to be about $3 \mathrm{~kJ} \mathrm{~mol}^{-1} \AA^{-2}$, with the main contribution coming from the dispersion term. The calculated phonon spectrum revealed two IRA-active vibrational modes (Fig. 7). Clearly, not only the positions, but also the relative intensities of the IR signals are in nice agreement with the experimental data. The band at $1296 \mathrm{~cm}^{-1}$ combines an in-phase asymmetric Si-O-Si stretching vibrations of the Si-O-Si linkage between two layers, while the band at $642 \mathrm{~cm}^{-1}$ involves symmetric Si-O-Si stretching vibrations of bonds oriented nearly parallel to the surface.

It is noteworthy that the bilayer structures were also observed by two depositionoxidation steps with one monolayer each. This implies that the monolayer is transformed into the bilayer silicate via breaking Si-O-Ru bonds and concurrent formation of the Si-O-Si linkages. Apparently, such a process is a thermodynamically unfavorable in the case of the Mo(112) support, that may explain why the formation of double-layer films has never been observed on Mo(112).

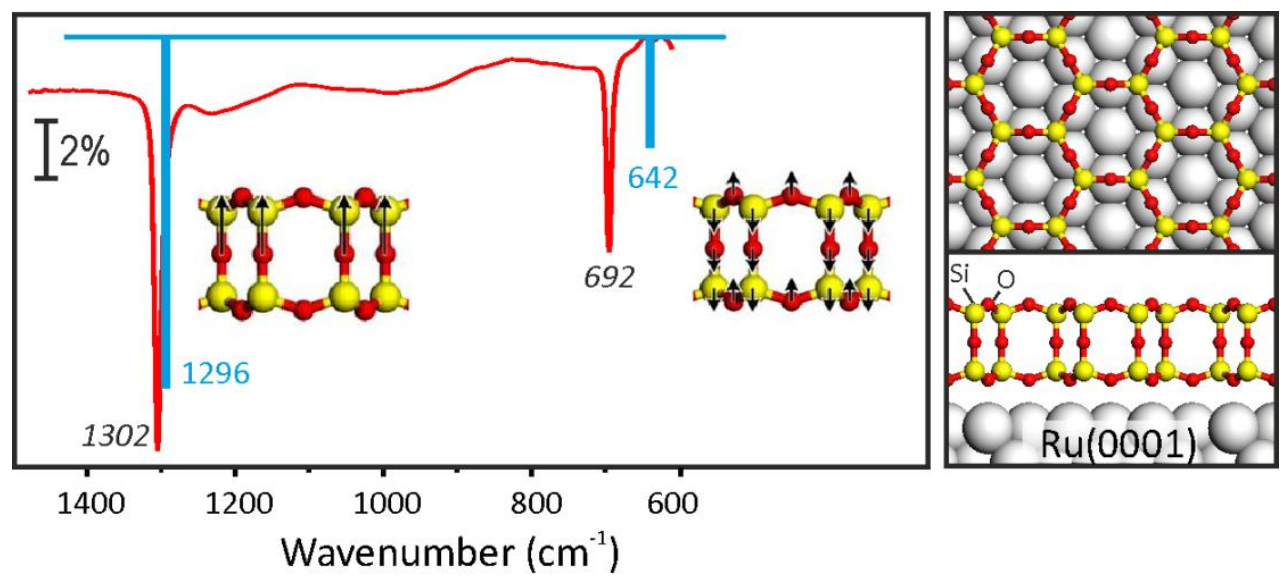

Figure 7. Experimental (in red) and DFT-derived (in blue) IRA spectra of the double-layer silicate film on Ru(0001). The corresponding vibrational modes are schematically shown as insets.

Having double-layer silicate films grown on $\mathrm{Ru}(0001)$, it was near at hand to prepare multilayer films in a layer-by-layer mode. However, growth of silicate films composed of bilayer 
sheets yet remains unsuccessful. For instance, the silica films possessing $\mathrm{Si}$ in amounts sufficient for the formation of four silicate layers showed a smooth, although not atomically flat surface [55]. As in the case of mono- and bilayer structures, the substantial changes were observed by IRAS. The intensities of the 1300 and $694 \mathrm{~cm}^{-1}$ bands strongly attenuated, while a new band developed at $1257 \mathrm{~cm}^{-1}$ with a prominent shoulder at $1164 \mathrm{~cm}^{-1}$. The latter spectrum is welldocumented for bulk-like silica [61, 62], indicating that thicker films exhibit a three-dimensional network of $\left[\mathrm{SiO}_{4}\right]$ tetrahedra rather than the sheet-like structure.
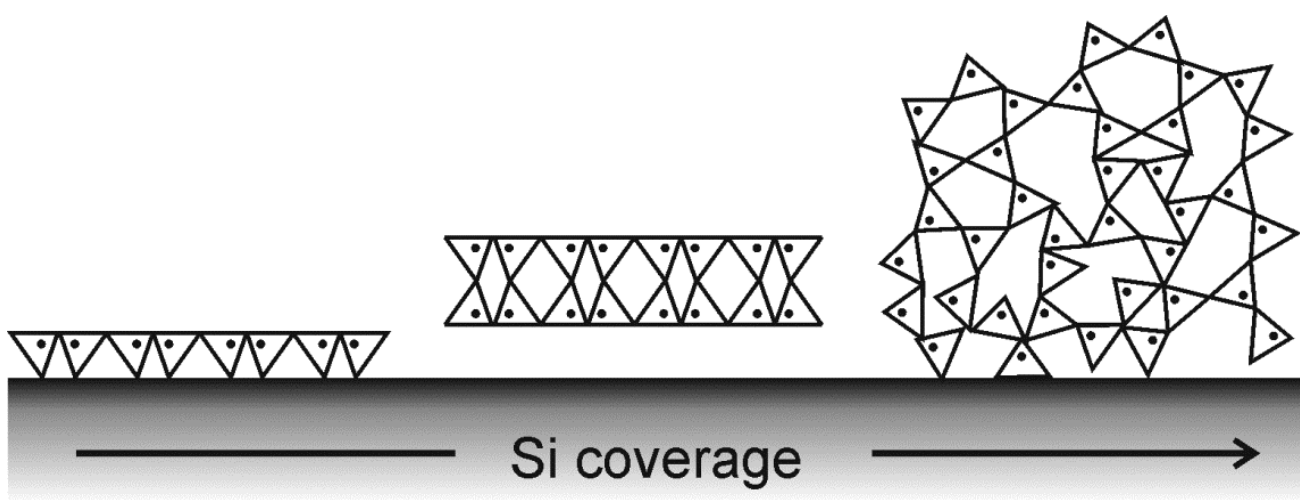

Figure 8. Principal structure of silicate films grown on $\mathrm{Ru}(0001)$ as a function of amounts of $\mathrm{Si}$ deposited prior to high-temperature annealing in $10^{-6} \mathrm{mbar}$ of $\mathrm{O}_{2}$.

Therefore, growth of silicate films on $\mathrm{Ru}(0001)$ as a function of Si coverage can be viewed as follows (Fig. 8). At low amounts of $\mathrm{Si}$, silica grows as a single-layer silicate. At increasing Si coverage, a double-layer silicate sheet is formed. At medium coverages, both structures coexist [55]. At higher amounts of $\mathrm{Si}$, the resulted films become virtually identical to the $\mathrm{SiO}_{2}$ films thermally grown on $\mathrm{Si}$ crystal surfaces. The double-layer films may exist in the crystalline and amorphous ("vitreous") phases often coexisting [63, 64], and as such they provide new opportunities for real space imaging of still poorly understood crystal-to-glass transitions. (This and related issues have been covered in our previous review papers [6, 9], and will not be discussed here). It is noteworthy that, at low coverages, silica prefers to form a single-layer closed film rather than the islands of a double-layer silicate. To some extent, this finding can be rationalized in terms of a definitely lower surface energy of siloxane (Si-O-Si)terminated silica when compared to Ru(0001). On the other hand, wetting of a metal substrate by oxide layer also implies relatively high adhesion energy, which is, in our case, primarily determined by the interfacial Si-O-Metal bonds. Indeed, as mentioned in the previous section, silicate films grown on Pt(111) only showed double-layer structures [56]. Moreover, the silicate films on $\mathrm{Pt}(111)$ are so far found solely in amorphous state, again indicating a critical role of a metal support in the atomic structure of silicate films. 
Altman et al. [65] have recently addressed the role of metal support by revisiting silica film preparation on the $\mathrm{Pd}(100)$ surface, previously introduced by Zhang et al. [66]. The authors observed hexagonal LEED patterns with a periodicity twice that of the substrate and with one of the overlayer close-packed directions paralleling Pd[011]. STM images observed crystalline domains which were limited to about five unit cells along two of the three main crystallographic directions but about tens of repeat units along the third one. The authors proposed the model, depicted in Fig. 9, assuming the crystalline bilayer structure of silica, where the regular domain boundaries help relieve stress caused by the lattice mismatch, i.e. in the same manner as observed for ultrathin alumina films on $\mathrm{NiAl}(110)$ [67]. DFT calculations on freestanding bilayers were performed to rationalize the effect of the substantial strain on the structure of the resulting films. The DFT results showed that relaxation orthogonal to the commensurate direction reduces the strain energy. As a consequence, the square substrate promotes epitaxial growth of crystalline $\mathrm{SiO}_{2}$ by providing an incommensurate direction along which the film can relax.

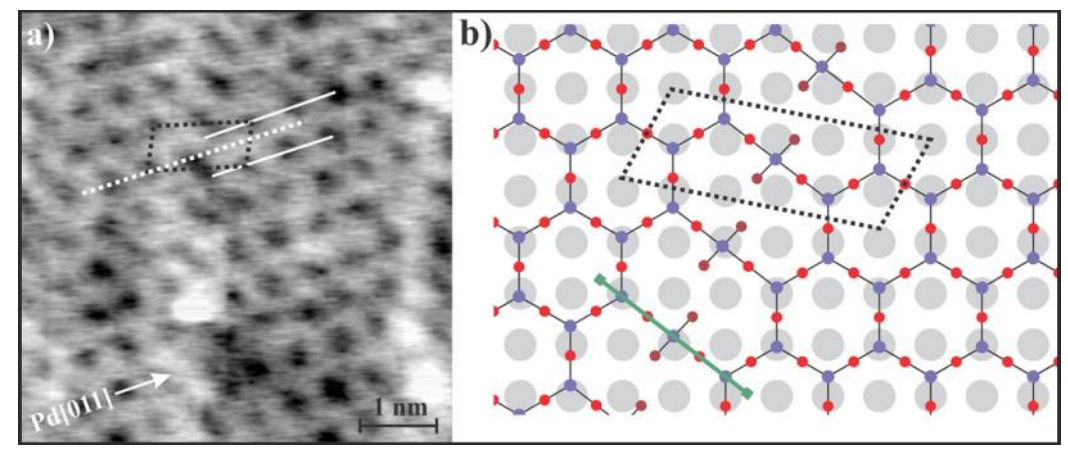

Figure 9. (a) STM image of a silica film grown on $\mathrm{Pd}(100)$ highlighting an antiphase domain boundary (ADB). The white lines show the offset between the rows across the APB while the black parallelogram connects the first repeat units on each side of the ADB. (b) Structural model of the ADB. Gray circles represent Pd, red circles $O$, and blue circles $\mathrm{Si}$; the darker red circles highlight $\mathrm{O}$ atoms at a lower depth. The dotted parallelogram connects the centers of the six-membered rings on either side of the $A D B$, which are assigned to the prominent dark spots in the STM images. Reprinted with permission from ref. [65]. Copyright (2013). American Chemical Society.

To conclude this section, we notice that the double-layer structure of silicate films grown on noble metal surfaces resembles unbranched zweier double layer described for the hexacelsian-type high temperature silicates of feldspar composition, for example, $\mathrm{Ba}\left[(\mathrm{AlSi}) \mathrm{O}_{4}\right]_{2}(\mathrm{hT})$ (see Fig. 7.25 in ref. [1]). However, in these compounds, half of the silicon atoms are replaced by aluminum atoms, and the negatively charged double layers are held together by cations such as $\mathrm{Ba}, \mathrm{Ca}$, or $\mathrm{Sr}$. 


\section{Hydroxylation of silicate films}

The interaction of silica with water plays an important role in natural processes such as weathering and dissolution. Also, natural silica-based minerals contain considerable amounts of water. In addition, the catalytic performance of silica, either as a support or an active phase, is primarily determined by hydroxyl species, which are commonly discussed in terms of isolated silanols, i.e. single silanols $(\mathrm{Si}-\mathrm{OH})$, geminal silanols $\left(\mathrm{Si}-(\mathrm{OH})_{2}\right)$, and groups of hydrogen-bonded silanols [5].

It is generally accepted in the literature that silica hydroxylation proceeds via "opening" of asymmetrically strained siloxane bridge sites or edge-shared tetrahedral dimers as shown schematically below:

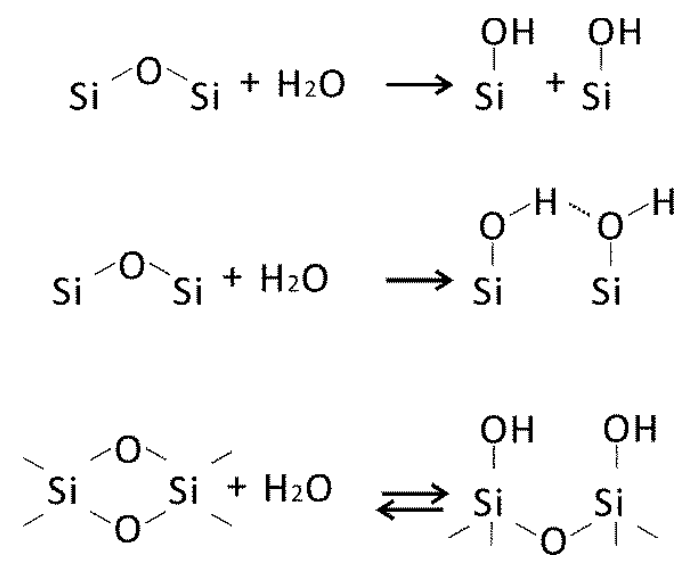

Subsequent water then adsorbs on the silanol covered surface rather than the pristine siloxane surface. The majority of the studies on this subject have been carried out on high surface area silica samples synthetized using "wet chemistry", primarily by dehydration of silica gels at high temperatures, although some results indicated that such strained siloxanes may even be present on initially dry silica [68].

First studies on the interaction of water with single-layer silicate films on $\mathrm{Mo}(112)$ only showed the growth of three-dimensional water clusters at low temperatures [69]. No evidence for dissociation of water was found in our own studies as well [70]. However, the results indicated the formation of a crystalline ice film at $140-150 \mathrm{~K}$ showing a $\mathrm{c}(2 \times 2)$ superstructure with respect to the silicate layer. Such an epitaxial growth of ice on silicate is probably favored by the small lattice mismatch. Indeed, the O-O distance projected onto the (0001) plane of the ice Ih phase is $2.61 \AA$ fits fairly well the $2.73 \AA$ for the O-O distance in the topmost O-layer of the silicate. The proposed model consisted of a water bilayer similar to that considered for adsorption on metal surfaces, but the model remains to be verified by theoretical calculations. In principle, non-dissociative interaction of water with monolayer silicates can be explained by a strong film bonding to a metal and high degree of crystallinity. As the double-layer films are 
only weakly bound to the metal substrate, and exhibit more structural flexibility than the monolayer versions and a broad distribution of $\mathrm{N}$-membered rings, one would expect to see substantial differences in reactivity towards water.

Adsorption of deuterated water $\left(\mathrm{D}_{2} \mathrm{O}\right)$ onto double-layer films at $300 \mathrm{~K}$ did not result in any hydroxo species as judged by IRAS. To increase the sticking coefficient, water adsorption was performed at low temperatures ( $100 \mathrm{~K})$, resulting in an "amorphous solid water" (ASW) film (or ice, for simplicity). This ice overlayer sublimes at $\mathrm{T} \sim 160 \mathrm{~K}$, upon which hydroxo species are formed with a characteristic IRA signal peaked at $2765 \mathrm{~cm}^{-1}$ (Fig. 10a) which can straightforwardly be assigned to $v(O-D)$ stretching vibrations in isolated silanols [71]. These OD species only disappears on heating to elevated temperatures ( $1000 \mathrm{~K})$ via water desorption, indicating their high thermal stability. From the intensity of the corresponding TPD and IRAS signals, we estimate $\sim 0.1 \mathrm{~nm}^{-2}$ as an upper limit for the surface density of silanols which approximately corresponds to one $\mathrm{OH}$ (OD) per about 40 six-membered rings. This small concentration points to that hydroxylation under these conditions occurs primarily on defects rather than regular sites. Analysis of defect structures present in the silicate films has suggested step edges, exposing low-coordinated surface atoms, as the most plausible candidates for active sites for water dissociation. Such step edges (either between two silica terraces or along the border of "holes" in the films), in the absence of any reconstruction, must expose very reactive dangling Si-O bonds. Unfortunately, direct visualization of hydroxyls on double-layer films with STM was unsuccessful. Nonetheless, STM images of hydroxylated single-layer silicate surfaces, revealed surface species decorating steps and other line defects, whereas the regular sites are apparently free of hydroxyls (Fig. 10b).

Following the above-shown schemes of silica hydroxylation, two $\mathrm{OH}$ species must be formed: one includes oxygen from water $\left(\mathrm{O}_{\mathrm{w}}\right)$ and another one incorporates oxygen from silica $\left(\mathrm{O}_{\mathrm{s}}\right)$. To discriminate these species, isotopic experiments were carried out [71]. Figure 10c compares IRA-spectra of two silica films prepared with ${ }^{18} \mathrm{O}$ and ${ }^{16} \mathrm{O}$, respectively, both hydroxylated with ${ }^{16} \mathrm{O}$-labeled water. Clearly, the majority of hydroxo-species is originated from incoming water molecules. The reason, why a higher IR intensity is observed for ${ }^{16} \mathrm{O}_{w} \mathrm{D}$ species (at $2766 \mathrm{~cm}^{-1}$ ) when compared to ${ }^{18} \mathrm{O}_{\mathrm{s}} \mathrm{D}$ one (at $2743 \mathrm{~cm}^{-1}$ ), is still not well understood. In principle, this could be explained by: (i) the hydroxylation of metal-supported silicate films proceeds by way of a mechanism other than that commonly considered for three-dimensional silicas; (ii) the ${ }^{18} \mathrm{O}_{s} \mathrm{D}$ bond is oriented almost parallel to the surface and as such becomes nearly invisible in IRAS due to the metal selection rule, which states that only vibrational modes which give rise to an oscillating dipole normal to the metallic surface are active. DFT calculations remain to be done to understand these experimental findings. 

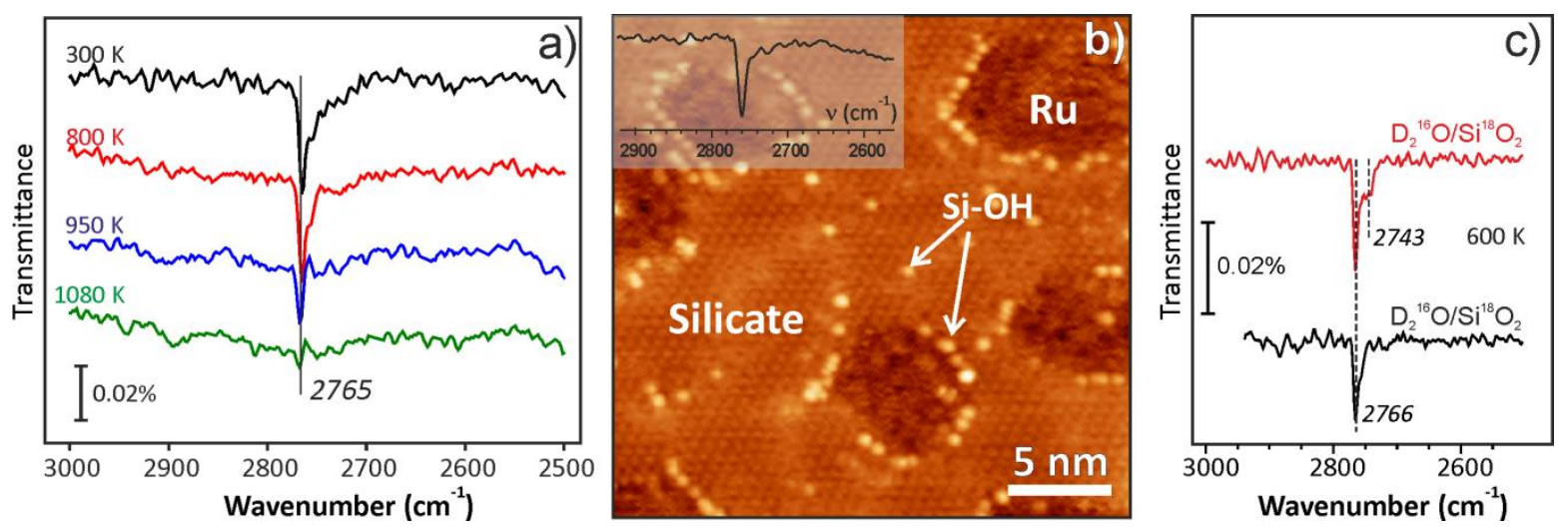

Figure 10. a) IRA-spectra of a hydroxylated double-layer silicate film on $\mathrm{Ru}(0001)$ heated stepwise to the temperature as indicated. b) STM image of a hydroxylated single-layer silicate film on Ru(0001) showing atomic size bright protrusions assigned to silanols on the basis of IRA-spectrum shown in the inset. c) Comparison of the IRA spectra of the double-layer silicate films, grown with ${ }^{16} \mathrm{O}$ and ${ }^{18} \mathrm{O}$ isotopes on $\mathrm{Ru}(0001)$ and hydroxylated with ${ }^{16} \mathrm{O}$ - labeled deuterated water, both heated to $600 \mathrm{~K}$.

\section{Substituted silicate films}

\section{Aluminosilicates}

Tetrahedrally coordinated $\mathrm{Si}$ in silicates may be substituted by other atoms. These atoms are commonly referred to as T- (after "tetrahedral") atoms regardless of their chemical nature. The feldspars, the most abundant minerals in the earth's crust, are constructed from a three-dimensional network of $\left[\mathrm{TO}_{4}\right]$ tetrahedra which can be described as a loop-branched dreier framework [1]. The excess negative charge resulted from the $\mathrm{Si}^{4+}$ substitution by $\mathrm{Al}^{3+}$ is balanced by positive ions, such as $\mathrm{H}^{+}$or alkali-metal cations. In the alkaline earth feldspars, each $\left[\mathrm{AlO}_{4}\right]$ tetrahedron is corner-linked to four $\left[\mathrm{SiO}_{4}\right]$ tetrahedra and vice versa, thus resulting in an ordered $\mathrm{Al} / \mathrm{Si}$ distribution among the available T-sites. This is in agreement with the so called Loewenstein's rule [72] stating from the electrostatic grounds that Al-O-Al linkages in aluminosilicates are forbidden. Zeolites are microporous members of the aluminosilicate family and play an important role in chemical industry. More than 40 naturally occurring zeolite frameworks are identified [73], and millions of hypothetical structures have been predicted based on topological considerations [74].

Since early days of "surface science" there were attempts to prepare adequate models to study chemical reactions on zeolites using surface sensitive tools. The first preparation of such model seems to be referred to the work of Somorjai and co-workers [75], who grew thin films ( $<10 \mathrm{~nm}$ ) of silica-alumina by argon ion beam sputter deposition on metal (gold) foil using zeolites as targets. Structural characterization showed that the prepared films were 
homogeneous and amorphous. Goodman's group [76] used Al deposition onto an amorphous $\mathrm{SiO}_{2}$ film support to prepare mixed $\mathrm{Al}_{2} \mathrm{O}_{3} / \mathrm{SiO}_{2}$ thin films. Although the results indicated that the electronic structures of the films were very similar to that of bulk aluminosilicates, the resulted films were poorly defined to establish structure-property relationships on zeolitic surfaces.

Certainly, following successful preparation of a crystalline silicate film on $\mathrm{Mo}(112)$, it was near at hand to use it as a template for growth of well-ordered aluminosilicate films. The preparation, including Al deposition onto the preformed single layer silicate film, resulted in disordered films. To facilitate the intermixing of $\mathrm{Al}$ and $\mathrm{Si}$ atoms in the film, their co-deposition onto $\mathrm{Mo}(112)$ in oxygen ambient was used, followed by annealing at $1100 \mathrm{~K}$ [77]. Structural characterization of the films at low $\mathrm{Al} / \mathrm{Si}$ ratios showed only small changes when compared to a pure silicate film. However, STM images revealed additional atomic features, whose density correlated well with the Al content in the film. The random distribution of these Al-related species suggested a random distribution of the Al ions in the film. However, determination of the atomic structure of an Al-substituted silicate film was only possible after DFT calculations of Sauer and co-workers who suggested the model shown in Fig. 11. In this model, Al is only surrounded by three oxygen ions. These $\left[\mathrm{AlO}_{3}\right]$ units are linked to $\left[\mathrm{SiO}_{4}\right]$ tetrahedra by sharing one oxygen. Certainly, a metal support is strongly involved to have such a structure stable.

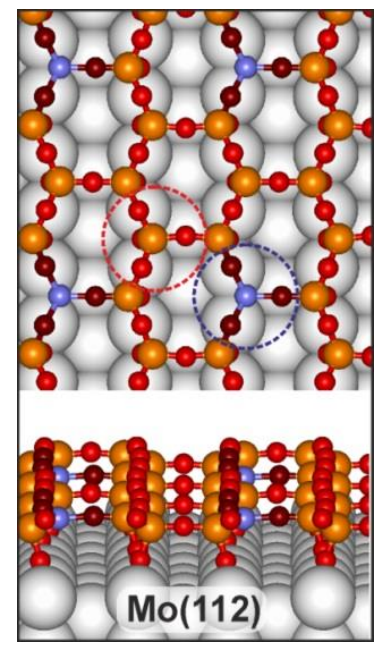

Figure 11. Structural model (in top and perspective views) of a single-layer aluminosilicate film on Mo(112). The $\left[\mathrm{SiO}_{4}\right]$ and $\left[\mathrm{AlO}_{3}\right]$ building units are highlighted by red and blue circles, respectively.

In the next step, the aluminosilicate films were prepared on $\mathrm{Ru}(0001)$ in a similar way, i.e. by $\mathrm{Si}$ and $\mathrm{Al}$ deposition in oxygen ambient (in total amounts equal to the amount of $\mathrm{Si}$ necessary to prepare a pure double-layer silicate film) and subsequent high temperature annealing [78]. In contrast to the random distribution of Al observed in single-layer films on $\mathrm{Mo}(112)$, the double-layer aluminosilicate films on $\mathrm{Ru}(0001)$ at low Ai:Si atomic ratios exhibited segregation into Al-rich and all-Si domains (Fig. 12). To some extent, this finding contradicts 
common view that the Al atoms arrange in zeolites as far as possible from each other [79]. To some extent, this effect can be explained by the lattice strain associated with the Al incorporation into the silicate frame, which can be minimized if Al-containing species locate near each other as theoretically predicted [80].

At low $\mathrm{Al} / \mathrm{Si}$ ratios, the $\mathrm{Al}$ ions seem to be preferentially located in the bottom layer, which is closer to the metal substrate that behaves as an electron reservoir to compensate charge imbalance caused by the substitution. However, following the Loewenstein's rule, at molar ratios above 0.5 , Al starts to occupy the cationic sites in the upper layer. Such films showed a rather uniform contrast in STM (the domain structure could not be distinguished anymore), although the surface exposed both crystalline and disordered phases.

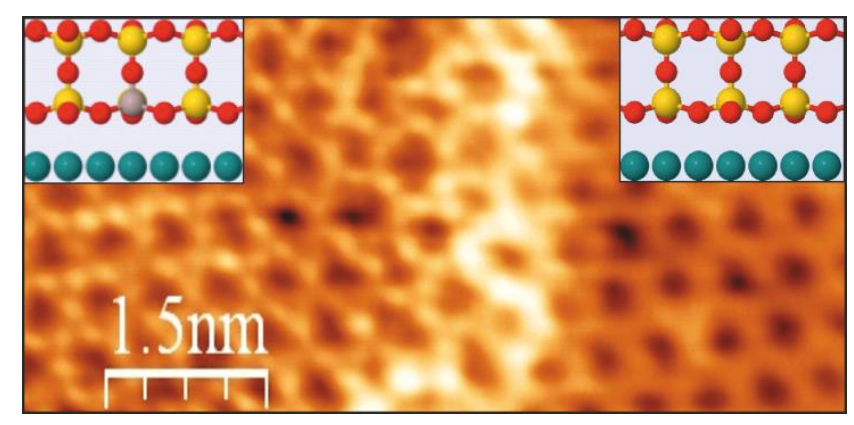

Figure 12. High-resolution STM image of an aluminosilicate film on Ru(0001) at low Al:Si (1:5) ratio. Two domains are seen: The Al-rich (in the left portion of the image) and the "all-Si" film (on the right). Structural models in the cross view are shown as insets.

The presence of $\mathrm{Al}$ in the top layer at high $\mathrm{Al} / \mathrm{Si}$ ratios is evidenced by hydroxylation experiments. Water adsorption at $\sim 100 \mathrm{~K}$ and subsequent heating to $300 \mathrm{~K}$ results in a sharp IRAS signal at $3594 \mathrm{~cm}^{-1}$, which falls in the frequency range of the hydroxyl groups in the bridging $\mathrm{Si}-(\mathrm{OH})_{b r}$-Al positions in zeolites [81]. To compare, only silanol (Si-OH) groups at 3750 $\mathrm{cm}^{-1}$ are observed in IRA-spectra of pure silicate films (see the previous section). The bridging hydroxyls are thermally stable up to $\sim 650 \mathrm{~K}$. Once formed $\mathrm{OH}$ groups can be replaced by OD upon exposure to $\mathrm{D}_{2} \mathrm{O}$ [78], thus indicating $\mathrm{H}-\mathrm{D}$ exchange reaction, which is a well-known phenomenon in zeolite chemistry.

The acidity of the prepared aluminosilicate films was measured by adsorption of weak bases, such as $\mathrm{CO}$ and ethylene, and strong bases such as ammonia and pyridine [82]. For example, $\mathrm{CO}$ binds to the acidic proton through the $\mathrm{C}$ atom to form an $\mathrm{CO}$ ' $\mathrm{HO}$ adduct. This, in turn, induces a red-shift of the $\mathrm{OH}$ stretching frequency, $v(\mathrm{OH})$, as well as a blue-shift of $v(\mathrm{CO})$ (when compared to $\mathrm{CO}$ in a gas phase, i.e. $2143 \mathrm{~cm}^{-1}$ ). The magnitude of the shift is proportional to the degree of acidity $[83,84]$. The observed red-shifts about 379 and $243 \mathrm{~cm}^{-1}$, for $v(O H)$ and $v(O D)$, and the blue-shift of $40 \mathrm{~cm}^{-1}$ for $v(C O)$, respectively, suggested that these films exhibit one of the highest acidity ever reported for zeolites [78]. 
All in all, the characteristics of the metal supported aluminosilicate films possessing $\mathrm{Si}$ $\mathrm{OH}-\mathrm{Al}$ surface species fit well into what is known about regular zeolitic surfaces. These films constitute the first well-defined model system where the surface properties of zeolites can be modeled by a surface-science approach $[8,85]$.

\section{Fe-silicates}

We now address the substitution patterns observed for transition metals. Zeolites containing $3 \mathrm{~d}$ metals are commonly used as catalysts. For example, Fe-zeolites and Fe-silicalites efficiently catalyse several industrially important oxidation reactions [86, 87]. These materials are formed by substitution of a small fraction of $\mathrm{Si}^{4+}$ with $\mathrm{Fe}^{3+}$ in the framework. Also clay minerals often contain redox-active $\mathrm{Fe}^{3+}$ species in the octahedral sheet, which participate in electron transfer reactions and play an important role in biogeochemical processes $[88,89]$.

Fe-doped silicate films were prepared on $\mathrm{Ru}(0001)$ in the same way as aluminosilicates, i.e. by Si and Fe deposition in oxygen ambient and high-temperature annealing [13]. Figure 13 collects IRA spectra observed on Fe-silicate films at increasing Fe:Si ratio as measured by XPS. Clearly, the $1300 \mathrm{~cm}^{-1}$ and $692 \mathrm{~cm}^{-1}$ bands gradually attenuate and ultimately disappear at Fe:Si $=1$, whereas a sharp and strong band at $1005 \mathrm{~cm}^{-1}$ together with a weak band at $674 \mathrm{~cm}^{-1}$ appear and gain intensity with increasing Fe content. Obviously, the Si-O-Si linkage between top and bottom silicate layers is no more present in the Fe-silicate film at the 1:1 ratio. Such a spectral evolution is characteristic for a two-component system, that suggests spatial segregation into the Fe-containing and pure silica phases rather than a uniform distribution of Fe in the silicate framework. For comparison, Al incorporation in silicate films only causes a red shift and broadening of the principal phonon bands (e.g. from 1300 to $1270 \mathrm{~cm}^{-1}$ ) $[78,85]$. The STM images as well as LEED patterns of the Fe-containing films (insets in Fig. 13) revealed the unit cell rotated by $30^{\circ}$ with respect to $\mathrm{Ru}(0001)$, and the lattice constant shortened to about $5.25 \AA$ ( $c f 5.42 \AA$ in the pure silicate film). In addition, flower-like satellite spots clearly indicate a Moiré structure which is commonly observed for interfaces having a lattice mismatch. 


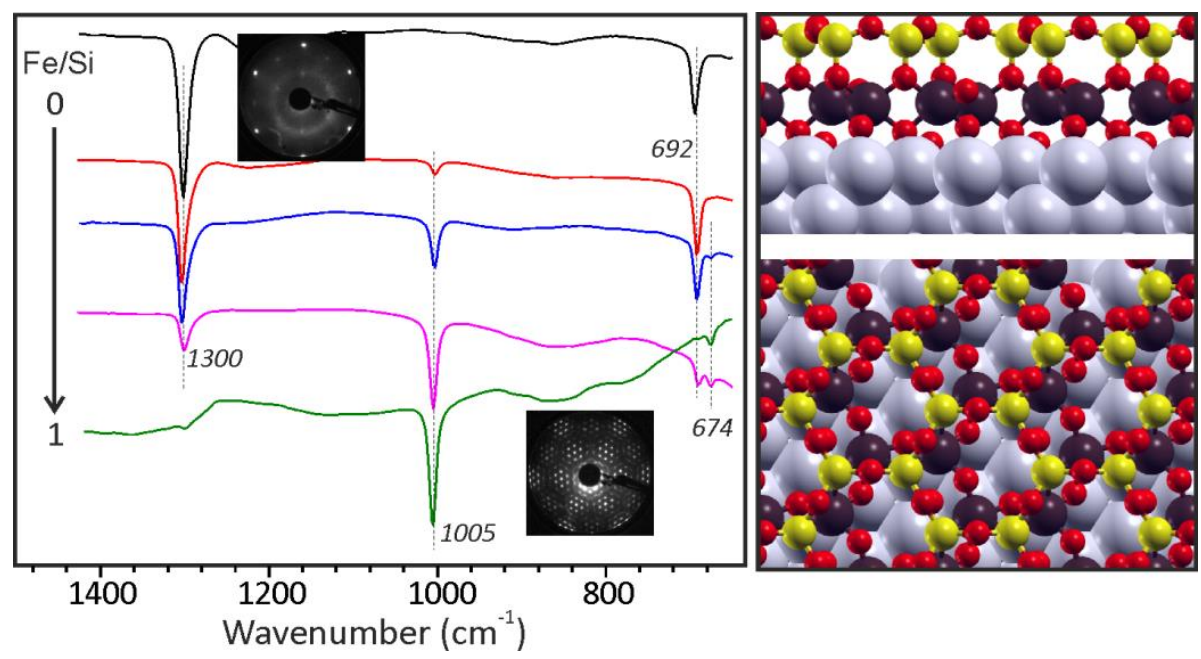

Figure 13. (Left) IRA-spectra of the Fe-substituted double-layer silicate films grown on Ru(0001) at increasing Fe:Si ratio as indicated. LEED patterns of pure silicate and Fe-silicate films are shown as insets. (Right) Structural model (top and cross views) of the Fe-silicate film at Fe:Si = 1:1 (see text).

DFT simulations were crucial in establishing the atomic structure of the Fe-doped silicate films [13]. Although the experimentally observed film rotation with respect to $\mathrm{Ru}(0001)$ was not included in the calculations, the structural model shown in Fig. 13 explains basically all experimental findings. In this structure, all Fe cations are octahedrally coordinated, and they constitute the bottom layer, while a silicate layer remains on top. The strongest IRA signal was computed at $1002 \mathrm{~cm}^{-1}$, which is in excellent agreement with the experimental value $1005 \mathrm{~cm}^{-1}$, and assigned to stretching of the $\mathrm{Si}-\mathrm{O}$ bonds oriented perpendicular to the surface. The lower frequency band originates from the Si-O-Si bending, and therefore only slightly sensitive to the presence of $\mathrm{Fe}$ in the film. The DFT results also gave rationale to the observed phase separation. When compared to the random distribution of $\mathrm{Fe}$ in the Si sites, a considerable energy gain is obtained when all $\mathrm{Si}$ atoms in the bottom layer are substituted by Fe.

In fact, the structure depicted in Fig. 13 represents a single T-O sheet in clay minerals (see Fig. 1). Indeed, the structure bears close similarities to the mineral nontronite, one of the Fe-rich smectites. An ideal nontronite is formed by an Fe-hydroxide sheet placed between two silicate sheets. In essence, the prepared Fe-silicate film can be viewed as a single sheet of dehydroxylated nontronite [90], where one T-layer is replaced by a metal support.

Note again, that the Fe-silicate film is structurally very different from the aluminosilicate film, albeit similarly prepared. The difference between the structural motifs in Al- and Fesilicate films mimics the different behaviour of naturally occurring Al- and Fe-silicate materials. In aluminosilicate films, $\mathrm{Al}^{3+}$ is present in four-fold coordination typical of natural zeolites (ironzeolites are not found in nature), whereas the Fe-silicate film adapts the layered structure of iron oxide - silicate materials, characteristic for clay minerals. 


\section{Silicate films and graphene}

As previously mentioned, structural defects on single-layer silicate films on Mo(112) and $\mathrm{Ru}(0001)$ are mostly represented by domain boundaries. In particular on $\mathrm{Mo}(112)$, they form a line of alternating octagons and tetragons (or 8-and 4-membered rings, respectively) [45] (Fig. $5 a)$. Defect structures obtained on a Ru(0001) substrate are more complex, often showing a network of line defects characteristic for mosaic-like films (Fig. 5b). However, close inspection of STM images also revealed few "point" defects, as shown in Fig. 14a, which consist of a hexagon surrounded by 3 pentagons and 3 heptagons.
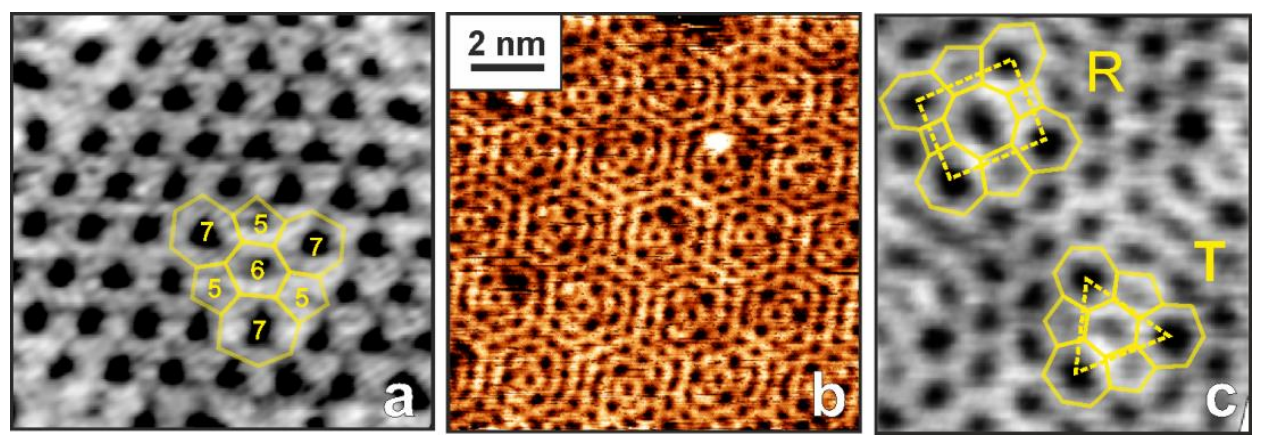

Figure 14. STM images of structural defects observed on single-layer silicate films on Ru(0001). (a) Single "blister". (b) Periodic defects observed after UHV annealing, which are zoomed in (c).

The number of such defects and its spatial distribution depend on preparation and change dramatically upon UHV annealing to $1100 \mathrm{~K}$ [14]. LEED and STM results showed that the UHVannealed film is azimuthally rotated by $30^{\circ}$ with respect to the $\mathrm{Ru}(0001)$ surface, thus resulting in shortening of the lattice constant to about $5.23 \AA$ and in Moiré-like structure. The IRAspectra showed some changes, although the principal (i.e., monolayer) structure of the film is certainly maintained. The STM image in Fig. 14b shows that the film is no longer represented by hexagons exclusively, but exhibits a 2D array of structural defects, marked as triangles (T) and rectangles $(R)$ in Fig. 14c, which are surrounded by a hexagonal network. The high-resolution STM images revealed that the T-defects are the same as observed, albeit rarely, in the original films (Fig. 14a). The R-defects constitutes an octagon surrounded by two pentagons, two tetragons and four heptagons, thus exhibiting the two-fold symmetry. The T-defects appear to be energetically more favorable than the R-defects as they dominate the surface after prolonged annealing. Importantly, films re-oxidation in $10^{-6} \mathrm{mbar}_{2}$ at $1100 \mathrm{~K}$ practically recovers the original structure of the film, thus suggesting that the formation of the patterned defects is thermodynamically driven process.

Literature survey revealed that similar defects has previously been considered for carbon nano-materials [91], in particular the T-defect on graphene is referred to as "blister" 
[92]. Since defects in graphene are expected to play a key role in the functional properties of working materials, various types of defects have been addressed both experimentally and theoretically (see recent reviews [93, 94]). Many defects in carbon sheets are described in terms of the Stone-Thrower-Wales [95, 96] (STW) defects (where four hexagons are converted into pentagon-heptagon pairs) which together with the so called "inverse STW" defects are considered as building blocks for a wide range of defect structures [97]. On the basis of DFT simulations, Lusk and Carr $[92,97,98]$ have recently suggested patterning the defects in the periodic structures as a tool to create artificial materials, that might exhibit entirely new properties. They demonstrated this approach by constructing 2D carbon allotropes referred to as haeckelites [91]. However, such hypothetical structures were not yet observed for graphene.

Obviously, a single silicate layer may be considered as a graphene composed of [ $\left.\mathrm{SiO}_{4}\right]$ entities instead of the $\mathrm{C}$ atoms. As such a single-layer silicate can be referred to as silicatene [14] similarly to graphene and Si-based analog called silicene [99, 100]. Similar to silicatene, silicene does not exist as a free-standing sheet and needs a support. However, in contrast to a low density of defects observed on silicene and graphene films grown on metal surfaces, silicatene turned out to show a variety of defect structures. Such a behavior may be resulted from the relatively high flexibility of the $\mathrm{Si}-\mathrm{O}$ bonds constituting a $\left[\mathrm{SiO}_{4}\right]$ unit, thus providing additional degree of freedom.

Driven primarily by electronic applications, there were few attempts to combine graphene and silica in one system. It was found that Si readily migrates through the graphene sheet and intercalates the graphene/metal interface [101-103]. Subsequent oxidation resulted in an amorphous thin silica film between the graphene and the metal as judged by XPS [104]. However, the atomic structure of the silica formed underneath the graphene remains, in essence, unknown. Oxidation of the graphene/silica/Ru films at higher temperatures, in attempt to make silica layer better ordered, would certainly result in burning graphene towards CO. Therefore, as alternative approach, we suggest to begin with a silicate film in order to grow graphene layer(s) underneath, aimed at the fabrication of silicate-graphene layered films [105].

Commonly, decomposition of ethylene or other unsaturated hydrocarbons is used for the preparation of graphene on metal surfaces. Direct exposure of the double-layer silicate film on $\mathrm{Ru}(0001)$ to ethylene at UHV-compatible pressures (typically, $10^{-6} \mathrm{mbar}$ ) causes no considerable changes in IRAS, XPS and LEED. Even subsequent UHV annealing showed no any indication for new structures. On the other hand, ethylene exposure at elevated temperatures $(\sim 1100 \mathrm{~K})$ resulted in partial or full reduction of silica to metallic-like Si. To reconcile these two limits, the film was exposed to high pressures at relatively low temperatures (in this case, 10 mbar $\mathrm{C}_{2} \mathrm{H}_{4}$ at $450 \mathrm{~K}$ ), which intercalate substantial amounts of carbonaceous species at the interface, but do not destroy the principal structure of the film. The samples were then annealed at $\sim 1100 \mathrm{~K}$ in UHV to make films well-ordered films as monitored by LEED. 


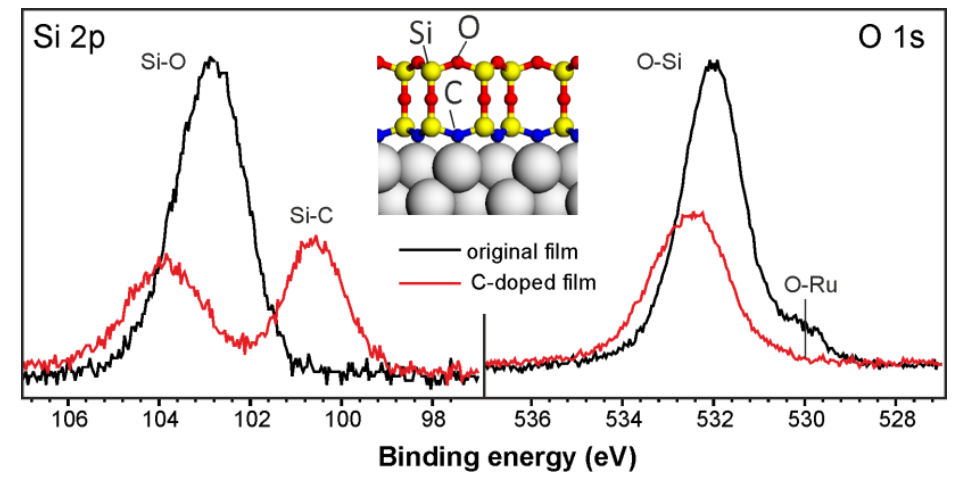

Figure 15. Si $2 p$ and $\mathrm{O}$ 1s regions in XP-spectra of pristine (black lines) and carbon-modified (red lines) silicate films. The structural model is shown in inset.

XPS study showed that the amount of oxygen is decreased by a factor of $\sim 2$, whereas the total amount of $\mathrm{Si}$ in the system remains the same, although it splits in two, almost equally populated species (Fig. 15). The high BE signal (at $104 \mathrm{eV}$ ) remains assigned to Si coordinated to oxygen ions, whereas the low BE signal at $\sim 101 \mathrm{eV}$ is characteristic for Si coordinated to $\mathrm{C}$ like in silicon carbide [106]. Therefore, the XPS results suggest that about a half of oxygen ions in the silicate film is replaced by carbon upon UHV annealing. The extent of such substitution depends on the ethylene exposure time. The proposed structure is depicted in Fig. 15, which can be considered as a silicatene placed on top of a single layer of silicon carbide formed on a metal support. There are obvious similarities between this structure and the one proposed for a silicate layer on $\mathrm{SiC}(0001)$ surface [36] (see Fig. 4). It is therefore plausible that the C atoms form chemical bonds to the Ru surface atoms and hence explain a much better film ordering observed by LEED. However, the DFT calculations remain to be done to verify the proposed model.

Finally, the results indicate that the fabrication of layered graphene/silicate structures on a metal substrate is hardly possible by synthesis based on the "intercalation" approach. Nonetheless, the resulted structure may be interesting for metal/oxide/semiconductor systems in nano-electronics.

\section{Concluding remarks}

The experimental results available to date show that silicate (i.e. a monolayer of cornersharing $\left[\mathrm{SiO}_{4}\right]$ tetrahedra) can be grown under certain conditions on many metal substrates. The principal structure of the films (single layer vs double-layer, crystalline vs amorphous) is basically governed by Si-O-metal bond strength: Metals with high oxygen adsorption energy favor the formation of perfect silicate films. Noble metals are prone for the formation of an amorphous, double-layer silicate sheet. The metals with intermediate energies may form either of the structures. 
All silicate films reported so far were experimentally observed only being supported on various solids. One may reasonably raise a question about whether it is possible to fabricate such films unsupported like, for example, graphene. Recently, Ciraci and co-workers addressed this issue from the DFT point of view [107]. The authors suggested a single-layer honeycomblike allotrope, $h \alpha$ silica in their notation, which is derived from the oxidation of silicene (i.e. a single layer of $\mathrm{Si}$ atoms), and has intriguing atomic structure with reentrant bond angles in hexagons. Also, the calculations predict interesting physical properties such as, for example, a negative Poisson's ratio and a high piezoelectric coefficient.

Upon introducing other metal in film preparation, well-defined substituted silicate films can be fabricated. In the case of Al, aluminosilicate films exposing zeolite-like, highly acidic surfaces are obtained. Growth of Fe-substituted silicate results in layered T-O sheets characteristic for clay minerals.

There are certain structural similarities observed between single-layer silicate and graphene, which allows us to call a metal supported single layer silicate as silicatene. Although, fabrication of free-standing silicatene remains challenging, preparation of silicatene-graphene hybrid structures on metal supports is tempting.

\section{Acknowledgements}

We thank all our co-workers, whose names appear in the cited papers, for their tremendous work in the laboratories. We gratefully acknowledge theory groups of Prof. Sauer (Humboldt University in Berlin) and Prof. Pacchioni (University Milano) for the fruitful collaboration. The work has been supported by Fonds der Chemischen Industrie and Deutsche Forschungsgemeinschaft through Collaborative Research Center SFB 1109, administered by TU Berlin.

\section{References}

1. Liebau, F., Structural Chemistry of Silicates. Structure, Bonding, and Classification. 1985: Springer.

2. Hattori, T., et al., Chemical and electronic structure of SiO2/Si interfacial transition layer. Applied Surface Science, 2003. 212-213(0): p. 547-555.

3. Helms, C.R. and E.H. Poindexter, The silicon-silicon dioxide system: Its microstructure and imperfections. Reports on Progress in Physics, 1994. 57(8): p. 791.

4. Legrand, A.P., ed. The Surface Properties of Silicas

1998, John Wiley and Sons Ltd: New York

465.

5. Zhuravlev, L.T., The surface chemistry of amorphous silica. Zhuravlev model. Colloids and Surfaces A: Physicochemical and Engineering Aspects, 2000. 173(1-3): p. 1-38. 
6. Shaikhutdinov, S. and H.-J. Freund, Ultrathin Silica Films on Metals: The Long and Winding Road to Understanding the Atomic Structure. Advanced Materials, 2013. 25(1): p. 49-67.

7. Muller, D.A., et al., The electronic structure at the atomic scale of ultrathin gate oxides. Nature, 1999. 399(6738): p. 758-761.

8. Shaikhutdinov, S. and H.-J. Freund, Metal-Supported Aluminosilicate Ultrathin Films as a Versatile Tool for Studying the Surface Chemistry of Zeolites. ChemPhysChem, 2013. 14(1): p. 7177.

9. Heyde, M., S. Shaikhutdinov, and H.J. Freund, Two-dimensional silica: Crystalline and vitreous. Chemical Physics Letters, 2012. 550(0): p. 1-7.

10. Loeffler, D., et al., Growth and Structure of Crystalline Silica Sheet on Ru(0001). Physical Review Letters, 2010. 105(14).

11. Roth, W.J., et al., Two-Dimensional Zeolites: Current Status and Perspectives. Chemical Reviews, 2014. 114(9): p. 4807-4837.

12. Dawson, C.J., et al., Low-Density, Low-Energy, Zeolites Assembled from Double-Layer Silica Sheets. Chemistry of Materials, 2013. 25(19): p. 3816-3821.

13. Włodarczyk, R., et al., Atomic Structure of an Ultrathin Fe-Silicate Film Grown on a Metal: A Monolayer of Clay? Journal of the American Chemical Society, 2013. 135(51): p. 19222-19228.

14. Yang, B., et al., Patterned Defect Structures Predicted for Graphene Are Observed on Single-Layer Silica Films. Nano Letters, 2013. 13(9): p. 4422-4427.

15. Pryde, A.K.A. and M.T. Dove, On the Sequence of Phase Transitions in Tridymite. Physics and Chemistry of Minerals, 1998. 26(2): p. 171-179.

16. Keen, D. and M. Dove, Local structures of amorphous and crystalline phases of silica, SiO2, by neutron total scattering. Journal of Physics: Condensed Matter, 1999. 11(47): p. 9263.

17. Rignanese, G.M., et al., First-principles molecular-dynamics study of the (0001) $\alpha$-quartz surface. Physical Review B, 2000. 61(19): p. 13250-13255.

18. Goumans, T.P.M., et al., Structure and stability of the (001) alpha-quartz surface. Physical Chemistry Chemical Physics, 2007. 9(17): p. 2146-2152.

19. Malyi, O.I., V.V. Kulish, and C. Persson, In search of new reconstructions of (001) alpha-quartz surface: a first principles study. RSC Advances, 2014. 4(98): p. 55599-55603.

20. Hirose, K., et al., Photoelectron spectroscopy studies of SiO2/Si interfaces. Progress in Surface Science, 2007. 82(1): p. 3-54.

21. Hiraiwa, A., Si-SiO2 Interface, Electronic Properties, in digital Encyclopedia of Applied Physics. 2003, WILEY-VCH Verlag GmbH \& Co KGaA.

22. Cabrera, N. and N.F. Mott, Theory of Oxidation of Metals. Rep. Prog. Phys., 1948. 12: p. 163-184.

23. Finster, J., et al., Interpretation of XPS core level shifts and structure of thin silicon oxide layers. Surface Science, 1985. 152-153(PART 2): p. 1063-1070.

24. Braun, W. and H. Kuhlenbeck, Chemical structure of ultrathin thermally grown oxides on a Si(100)-wafer using core level photoemission. Surface Science, 1987. 180(1): p. 279-288.

25. Grunthaner, P.J., et al., The localization and crystallographic dependence of Si suboxide species at the SiO2/Si interface. Journal of Applied Physics, 1987. 61(2): p. 629-638.

26. Ichimura, S., et al., Ultrathin SiO2 film growth on Si by highly concentrated ozone. Thin Solid Films, 2000. 377-378(0): p. 518-524.

27. Herbots, N., et al., The formation of ordered, ultrathin SiO2/Si(100) interfaces grown on (1×1) Si(100). Materials Science and Engineering B: Solid-State Materials for Advanced Technology, 2001. 87(3): p. 303-316.

28. Tanaka, N., et al., First observation of SiO2/Si(100) interfaces by spherical aberration-corrected high-resolution transmission electron microscopy. Journal of Electron Microscopy, 2003. 52(1): p. 69-73. 
29. Barski, A., et al., Epitaxial growth of germanium dots on Si(001) surface covered by a very thin silicon oxide layer. Applied Physics Letters, 2000. 77(22): p. 3541-3543.

30. Rochet, F., et al., Growth of epitaxial silica on vicinal Si(001) surfaces during thermal oxidation in O2. Philosophical Magazine Part B, 1989. 59(3): p. 339-363.

31. Tomoya, O., et al., First-principles study of the electronic structures and dielectric properties of the Si/SiO2 interface. Journal of Physics: Condensed Matter, 2007. 19(36): p. 365202.

32. Katsuhiro, K., O. Tomoya, and H. Kikuji, First-principles study on electronic structure of Si/SiO2 interface-Effect of interface defects on local charge density. Science and Technology of Advanced Materials, 2007. 8(3): p. 204.

33. Kovačević, G. and B. Pivac, Modeling the interface between crystalline silicon and silicon oxide polymorphs. physica status solidi (a), 2013. 210(4): p. 717-722.

34. Raynaud, C., Silica films on silicon carbide: a review of electrical properties and device applications. Journal of Non-Crystalline Solids, 2001. 280(1-3): p. 1-31.

35. Presser, V. and K.G. Nickel, Silica on Silicon Carbide. Critical Reviews in Solid State and Materials Sciences, 2008. 33(1): p. 1-99.

36. Bernhardt, J., et al., Epitaxially ideal oxide--semiconductor interfaces: Silicate adlayers on hexagonal (0001) and (0001-bar) SiC surfaces. Applied Physics Letters, 1999. 74(8): p. 10841086.

37. Lu, W., P. Krüger, and J. Pollmann, Atomic and electronic structure of silicate adlayers on polar hexagonal SiC surfaces. Physical Review B, 2000. 61(20): p. 13737-13744.

38. Weissenrieder, J., et al., Atomic Structure of a Thin Silica Film on a Mo(112) Substrate: A TwoDimensional Network of $\mathrm{SiO}_{4}$ Tetrahedra. Physical Review Letters, 2005. 95(7): p. 076103.

39. Kaya, S., et al., On the geometrical and electronic structure of an ultra-thin crystalline silica film grown on Mo(112). Surface Science, 2007. 601(21): p. 4849-4861.

40. $\mathrm{Xu}, \mathrm{X}$. and D.W. Goodman, New approach to the preparation of ultrathin silicon dioxide films at low temperatures. Applied Physics Letters, 1992. 61(7): p. 774-776.

41. $\mathrm{Xu}, \mathrm{X}$. and D.W. Goodman, The preparation and characterization of ultra-thin silicon dioxide films on a Mo(110) surface. Surface Science, 1993. 282(3): p. 323-332.

42. He, J.W., et al., X-ray photoelectron spectroscopic characterization of ultra-thin silicon oxide films on a $\mathrm{Mo}(100)$ surface. Surface Science, 1992. 279(1-2): p. 119-126.

43. Schroeder, T., et al., Morphological and electronic properties of ultrathin crystalline silica epilayers on a Mo(112) substrate. Physical Review B, 2002. 66(16): p. 165422.

44. Schroeder, T., et al., Epitaxial growth of SiO2 on Mo(112). Surface Review and Letters, 2000. 7(12): p. 7-14.

45. Todorova, T.K., et al., Atomic structure of a thin silica film on a Mo(112) substrate: $A$ combined experimental and theoretical study. Physical Review B, 2006. 73(16): p. 165414.

46. Schroeder, T., et al., Single crystalline silicon dioxide films on Mo(112). Solid-State Electronics, 2001. 45(8): p. 1471-1478.

47. Chen, M.S., A.K. Santra, and D.W. Goodman, Structure of thin $\mathrm{SiO}_{2}$ films grown on $\mathrm{Mo}(112)$. Physical Review B, 2004. 69(15): p. 155404.

48. Giordano, L., et al., Structure and vibrational spectra of crystalline SiO2 ultra-thin films on Mo(112). Surface Science, 2005. 584(2-3): p. 225-236.

49. Chen, M. and D.W. Goodman, The structure of monolayer SiO2 on Mo(112): A 2-D [Si-O-Si] network or isolated [SiO4] units? Surface Science, 2006. 600(19): p. L255-L259.

50. Seifert, J., D. Blauth, and H. Winter, Evidence for 2D-Network Structure of Monolayer Silica Film on Mo(112). Physical Review Letters, 2009. 103(1): p. 017601.

51. Kinoshita, T. and S. Mizuno, Surface structure determination of silica single layer on Mo(112) by LEED. Surface Science, 2011. 605(13-14): p. 1209-1213. 
52. Sierka, M., et al., Interplay between theory and experiment in the quest for silica with reduced dimensionality grown on a Mo(112) surface. Chemical Physics Letters, 2006. 424(1-3): p. 115119.

53. Martinez, U., L. Giordano, and G. Pacchioni, Tuning the work function of ultrathin oxide films on metals by adsorption of alkali atoms. The Journal of Chemical Physics, 2008. 128(16): p. 1647078.

54. Lu, J.L., et al., Formation of one-dimensional crystalline silica on a metal substrate. Surface Science, 2006. 600(13): p. L164-L168.

55. Yang, B., et al., Thin silica films on Ru(0001): monolayer, bilayer and three-dimensional networks of [SiO4] tetrahedra. Physical Chemistry Chemical Physics, 2012.

56. Yu, X., et al., Support effects on the atomic structure of ultrathin silica films on metals. Applied Physics Letters, 2012. 100(15): p. 151608-4.

57. Ben Romdhane, F., et al., In Situ Growth of Cellular Two-Dimensional Silicon Oxide on Metal Substrates. ACS Nano, 2013. 7(6): p. 5175-5180.

58. Ben Romdhane, F., et al., Solid-State Growth of One- and Two-Dimensional Silica Structures on Metal Surfaces. The Journal of Physical Chemistry C, 2014. 118(36): p. 21001-21005.

59. Stacchiola, D.J., et al., Growth of stoichiometric subnanometer silica films. Applied Physics Letters, 2008. 92(1).

60. Kuhlenbeck, H., S. Shaikhutdinov, and H.-J. Freund, Well-Ordered Transition Metal Oxide Layers in Model Catalysis - A Series of Case Studies. Chemical Reviews, 2013. 113(6): p. 3986-4034.

61. Queeney, K.T., et al., Silicon Oxidation and UItra-Thin Oxide Formation on Silicon Studied by Infrared Absorption Spectroscopy. physica status solidi (a), 1999. 175(1): p. 77-88.

62. Queeney, K.T., et al., Infrared spectroscopic analysis of an ordered Si/SiO2 interface. Applied Physics Letters, 2004. 84(4): p. 493-495.

63. Lichtenstein, L., et al., The Atomic Structure of a Metal-Supported Vitreous Thin Silica Film. Angewandte Chemie International Edition, 2012. 51(2): p. 404-407.

64. Huang, P.Y., et al., Direct Imaging of a Two-Dimensional Silica Glass on Graphene. Nano Letters, 2012. 12(2): p. 1081-1086.

65. Altman, E.I., et al., Growth and Characterization of Crystalline Silica Films on Pd(100). The Journal of Physical Chemistry C, 2013. 117(49): p. 26144-26155.

66. Zhang, Z., et al., Preparation and characterization of atomically flat and ordered silica films on a Pd(100) surface. Thin Solid Films, 2008. 516(12): p. 3741-3746.

67. Libuda, J., et al., Structure and defects of an ordered alumina film on NiAl(110). Surface Science, 1994. 318(1-2): p. 61-73.

68. Chiang, C.M., B.R. Zegarski, and L.H. Dubois, First observation of strained siloxane bonds on silicon oxide thin films. The Journal of Physical Chemistry, 1993. 97(27): p. 6948-6950.

69. Wendt, S., et al., The interaction of water with silica thin films grown on Mo(1\&\#xa0;1\&\#xa0;2). Surface Science, 2004. 565(2-3): p. 107-120.

70. Kaya, S., et al., Formation of an Ordered Ice Layer on a Thin Silica Film. The Journal of Physical Chemistry C, 2006. 111(2): p. 759-764.

71. Yang, B., et al., Hydroxylation of Metal-Supported Sheet-Like Silica Films. The Journal of Physical Chemistry C, 2013. 117(16): p. 8336-8344.

72. Loewenstein, W., The Distribution of Aluminum in the Tetrahedra of Silicates and Aluminates. American Mineralogist, 1954. 39(1-2): p. 92-96.

73. Atlas of Zeolite Framework Types. 6-th ed, ed. Ch. Baerlocher, W.M. Meier, and D.H. Olson. 2007: Elsevier.

74. Foster, M.D. and M.M.J. Treacy. A Database of Hypothetical Zeolite Structures. Available from: http://www.hypotheticalzeolites.net. 
75. Böszörményi, I., et al., Model silica-alumina acid catalysts for surface science and catalysis studies prepared by argon ion beam sputter deposition using HY-zeolite targets. Catalysis Letters, 1991. 10(5): p. 343-355.

76. Gründling, C., J.A. Lercher, and D.W. Goodman, Preparation of mixed A/2O3/SiO2 thin films supported on Mo(100). Surface Science, 1994. 318(1-2): p. 97-103.

77. Stacchiola, D., et al., Synthesis and Structure of Ultrathin Aluminosilicate Films. Angewandte Chemie International Edition, 2006. 45(45): p. 7636-7639.

78. Boscoboinik, J.A., et al., Modeling Zeolites with Metal-Supported Two-Dimensional Aluminosilicate Films. Angewandte Chemie International Edition, 2012. 51(24): p. 6005-6008.

79. Dempsey, E., Acid strength and aluminum site reactivity of Y zeolites. Journal of Catalysis, 1974. 33(3): p. 497-499.

80. Schroder, K.P. and J.C. Sauer, Preferred Stability of Al-O-Si-O-Al Linkages in High Silica Zeolite Catalysts- Theoretical Predcitionss Contrary to Dempseys Rule Journal of Physical Chemistry, 1993. 97(25): p. 6579-6581.

81. Bordiga, S., et al., Assessing the Acidity of High Silica Chabazite H-SSZ-13 by FTIR Using CO as Molecular Probe: Comparison with H-SAPO-34. The Journal of Physical Chemistry B, 2005. 109(7): p. 2779-2784.

82. Boscoboinik, J.A., et al., Interaction of Probe Molecules with Bridging Hydroxyls of TwoDimensional Zeolites: A Surface Science Approach. The Journal of Physical Chemistry C, 2013. 117(26): p. 13547-13556.

83. Busca, G., Spectroscopic characterization of the acid properties of metal oxide catalysts. Catalysis Today, 1998. 41(1-3): p. 191-206.

84. Zecchina, A., C. Lamberti, and S. Bordiga, Surface acidity and basicity: General concepts. Catalysis Today, 1998. 41(1-3): p. 169-177.

85. Boscoboinik, J.A. and S. Shaikhutdinov, Exploring Zeolite Chemistry with the Tools of Surface Science: Challenges, Opportunities, and Limitations. Catalysis Letters, 2014. 144(12): p. 19871995.

86. Panov, G.I., Advances in Oxidation Catalysis; Oxidation of Benzene to Phenol by Nutrous Oxide. CATTECH, 2000. 4(1): p. 18-31.

87. Zecchina, A., et al., Structure and nuclearity of active sites in Fe-zeolites: comparison with iron sites in enzymes and homogeneous catalysts. Physical Chemistry Chemical Physics, 2007. 9(27): p. 3483-3499.

88. Anke, N., S. Michael, and B.H. Thomas, Redox Properties of Structural Fe in Smectite Clay Minerals, in Aquatic Redox Chemistry. 2011, American Chemical Society. p. 361-379.

89. Alexandrov, V., et al., Electron Exchange and Conduction in Nontronite from First-Principles. The Journal of Physical Chemistry C, 2013. 117(5): p. 2032-2040.

90. Dainyak, L.G., et al., Interpretation of the nontronite-dehydroxylate Mössbauer spectrum using EFG calculations. European Journal of Mineralogy, 2006. 18(6): p. 753-764.

91. Terrones, H., et al., New metallic allotropes of planar and tubular carbon. Physical Review Letters, 2000. 84(8): p. 1716-1719.

92. Lusk, M.T. and L.D. Carr, Nanoengineering Defect Structures on Graphene. Physical Review Letters, 2008. 100(17): p. 175503.

93. Batzill, M., The surface science of graphene: Metal interfaces, CVD synthesis, nanoribbons, chemical modifications, and defects. Surface Science Reports, 2012. 67(3-4): p. 83-115.

94. Banhart, F., J. Kotakoski, and A.V. Krasheninnikov, Structural Defects in Graphene. ACS Nano, 2010. 5(1): p. 26-41.

95. Stone, A.J. and D.J. Wales, Theoretical studies of icosahedral C60 and some related species. Chemical Physics Letters, 1986. 128(5-6): p. 501-503. 
96. Thrower, P.A., ed. Chemistry and Physics of Carbon, ed. J.P.L. Walker. Vol. 5. 1969. 262.

97. Lusk, M.T., D.T. Wu, and L.D. Carr, Graphene nanoengineering and the inverse Stone-ThrowerWales defect. Physical Review B, 2010. 81(15): p. 155444.

98. Lusk, M.T. and L.D. Carr, Creation of graphene allotropes using patterned defects. Carbon, 2009. 47(9): p. 2226-2232.

99. Vogt, P., et al., Silicene: Compelling Experimental Evidence for Graphenelike Two-Dimensional Silicon. Physical Review Letters, 2012. 108(15): p. 155501.

100. Guzmán-Verri, G.G. and L.C. Lew Yan Voon, Electronic structure of silicon-based nanostructures. Physical Review B, 2007. 76(7): p. 075131.

101. Kaloni, T.P., et al., Mechanism of Si intercalation in defective graphene on SiC. Journal of Materials Chemistry, 2012. 22(44): p. 23340-23343.

102. Mao, J., et al., Silicon layer intercalation of centimeter-scale, epitaxially grown monolayer graphene on Ru(0001). Applied Physics Letters, 2012. 100(9): p. -.

103. Cui, Y., et al., An exchange intercalation mechanism for the formation of a two-dimensional Si structure underneath graphene. Nano Research, 2012. 5(5): p. 352-360.

104. Lizzit, S., et al., Transfer-Free Electrical Insulation of Epitaxial Graphene from its Metal Substrate. Nano Letters, 2012. 12(9): p. 4503-4507.

105. Yang, B., S. Shaikhutdinov, and H.-J. Freund, Ultrathin silicatene/silicon-carbide hybrid film on a metal substrate. Surface Science, 2015. 632(0): p. 9-13.

106. Kusunoki, I. and Y. Igari, XPS study of a SiC film produced on Si(100) by reaction with a C2H2 beam. Applied Surface Science, 1992. 59(2): p. 95-104.

107. Özçelik, V.O., S. Cahangirov, and S. Ciraci, Stable Single-Layer Honeycomblike Structure of Silica. Physical Review Letters, 2014. 112(24): p. 246803. 